\title{
Multiview Imaging with Real-Time Microwave Camera from Known Positions
}

\author{
Jaime Laviada, Fernando Las-Heras \\ Dept. Ingeniería Eléctrica \\ Universidad de Oviedo \\ 33205, Gijón, Spain \\ Email: [jlaviada] [flasheras]@tsc.uniovi.es
}

\author{
Mohammad T. Ghasr, Reza Zoughi \\ Applied Microwave Nondestructive Testing Laboratory (amntl) \\ Missouri University of Science and Technology \\ MO 65409, Rolla, USA \\ Email: [mtg7w6][Zoughi]@mst.edu
}

\begin{abstract}
This contribution shows the application of multiview processing technique to a recently-developed real-time $3 \mathrm{D} \mathrm{mi-}$ crowave camera. The camera, which operates based on efficient synthetic aperture radar (SAR) imaging techniques, provides a good trade-off between fast image production and image quality. These cameras, as it happens with standard optical cameras, provide a good image of a volume in front of their apertures, but they cannot provide information about the complete object under test. Furthermore, it is usually convenient to consider multiple view angles as the best quality image is achieved when the area to be image is parallel to the aperture (as the specular reflection is captured). In this paper, we illustrate how recently-developed techniques, inspired from computer vision approaches, can be used to merge multiple views to obtain a more complete image of an object.
\end{abstract}

\section{INTRODUCTION}

Electromagnetic imaging exploits the capability of electromagnetic waves to penetrate through visually opaque (dielectric) materials to provide information, which cannot be observed by the human eye or optical cameras. There are a number of approaches [1] to build this kind of images depending on the particular nature of the problem, the degree of accuracy and the required processing speed.

One of the most effective imaging techniques is based on synthetic aperture radar (SAR) processing, which has been successfully applied to different fields such as security [2] and non-destructive evaluation [3]. In this approach, several transmitters and receivers are placed in an aperture to illuminate an object and capture the reflected field, which is subsequently processed using Fast Fourier Transform (FFT), to provide the final image.

This approach provides imaging information about the object right in front of imaging aperture. However, portions of the object that are not illuminated by the imaging aperture will not be detected and imaged [4], similar to when using a conventional optical camera. Nevertheless, if multiple views are captured, then computer vision (CV) techniques can be employed to merge them into a more complete model [5].

Some of these techniques from $\mathrm{CV}$ have been recently adapted to SAR approaches to bypass the aforementioned problems [6]. Furthermore, these techniques are mainly developed for relatively small scanners, which can be arbitrarily moved, in contrast to bulky scanners, but they are only able to image a smaller volume as a consequence of their smaller size. In this contribution, these techniques are applied for the first time to a recently-developed real-time microwave camera [7] in place of the usual raster scanning [6]. This step provides an intermediate milestone towards the possibility of developing a portable free-hand scanner.

\section{Multiview Imaging with Portable Microwave CAMERA}

As an intermediate step before including an accurate positioning system, here we consider the real-time camera positions are a priori known. This enables us to first validate the quality of the potential images that could be produced by the complete scanner consisting of the microwave camera together with the positioning system.

The real-time microwave camera used here [7] provides a set of non-uniformly spaced samples, which does not correspond to a uniform grid as required by conventional SAR algorithms. Thus, the raw data are processed as discussed in [7], to provide a set of samples equally spaced along the aperture of the microwave camera. These data are acquired for a preset number of frequencies in the $20-30 \mathrm{GHz}$ range. Subsequently, they are processed by means of a range-migration algorithm based on FFTs [8] to estimate the reflectivity $\rho_{n}\left(\vec{r}_{n}^{\prime}\right)$, where $\vec{r}_{n}^{\prime}$ denotes the observation point expressed in the local coordinates system for the $n$-th position.

If the positions of the microwave camera are known, then this local reflectivity can be translated into a global coordinates system. However, if this position information is contaminated by an error in the order of a wavelength or more, as it is usually the case when considering photogrametry or similar approaches, then the preferred way to merge the local reflectivities is to resort to non-coherent approaches. In particular, most of problems [4] analyzed with SAR techniques try to find areas with high reflections, as they usually indicate of anomalies, and therefore, it is convenient to use an approach with capability to keep the maximum values to avoid missing areas corresponding to points of interest, as the one proposed in [4]:

$$
\Gamma_{n}(\vec{r})=\max \left\{\Gamma_{n-1}(\vec{r}), \rho_{n}(\vec{r})\right\}
$$

where $\Gamma_{n}(\vec{r})$ is the total reflectivity computed after updating with the local reflectivity corresponding to the $n$-th position. 
This total reflectivity can be represented as an image to provide a real-time updated image.

\section{RESULTS}

In order to obtain a number of known positions, the object under test (a torso-like geometry with a bar attached as shown in Fig. 1a) is placed at a turntable whose center is separated $29 \mathrm{~cm}$ from the microwave camera aperture (see Fig. 1b). Six different positions are obtained by rotating the turntable by a step of $10^{\circ}$. It is important to note that this process is virtually equivalent to moving the camera around the object. Fig. 2 depicts this setup including the global coordinate system used in this paper.

At each position, the camera provides an interpolated matrix of $66 \times 61$ samples, spaced by $2.75 \mathrm{~mm}$ and $2 \mathrm{~mm}$ for the $x$-axis and $y$-axis respectively, corresponding to points in the aperture along a regular grid [7]. For each point at the aperture grid, the data is sampled at 121 frequencies between $20 \mathrm{GHz}$ to $30 \mathrm{GHz}$ but only the lowest 100 frequencies are retained to take advantage of the band in which the best antenna matching is available [7].

Imaging results for incremental partial views, according to the update equation given in (1), are shown in Fig. 3. As additional views are added to the image data progressively (Figs. 3 (a)-(f) corresponding to 1-6 views) the geometry of the target, including the bar, becomes more evident.

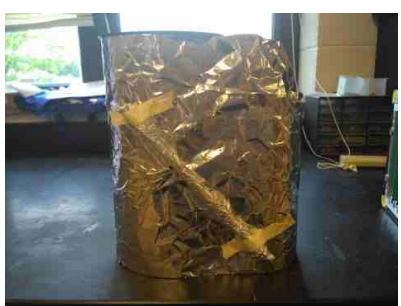

(a)

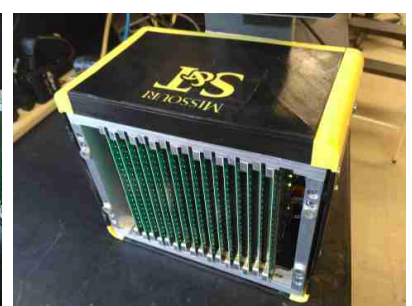

(b)
Fig. 1. Elements of the setup: (a) object under test, and (b) microwave camera from [7].

Fig. 2. Setup to acquire multiple views from controlled positions

\section{CONCLUSIONS}

The possibility of performing multiview imaging with a real-time microwave camera has been validated for the first time. The system, based on performing SAR acquisitions from different positions, shows the capability of merging the different views to achieve a complete three-dimensional model, which is not possible from a single view.

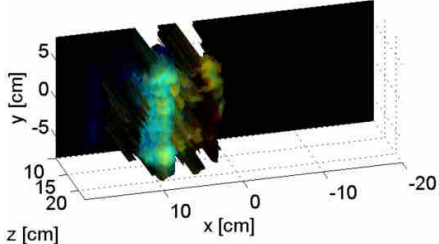

(a)

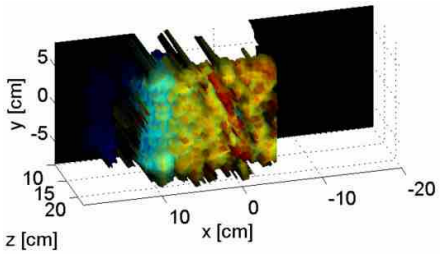

(c)

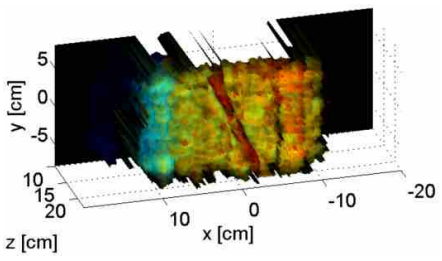

(e)

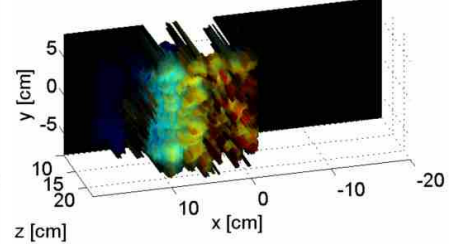

(b)

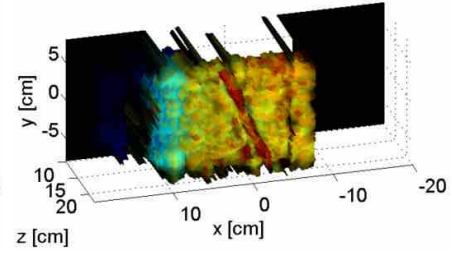

(d)

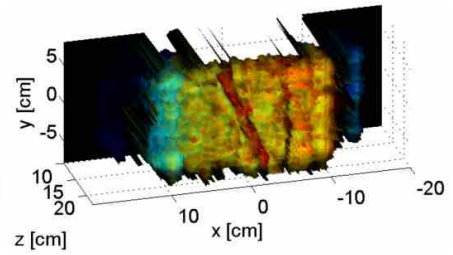

(f)
Fig. 3. Imaging results for incremental partial views: (a) 1 view, (b) 2 views, (c) 3 views, (d) 4 views, (e) 5 views, and (f) 6 views.

This approach supposes a middle step towards the possibility of achieving free-hand multiview scanning with portable cameras. For that purpose, efficient positioning algorithms are currently under study to complement the system with accurate real-time positioning.

\section{ACKNOWLEGMENT}

This work has been partially supported by the Ministerio de Ciencia e Innovación of Spain /FEDER under project TEC2014-55290-JIN.

\section{REFERENCES}

[1] M. Pastorino, Microwave Imaging. John Wiley \& Sons, Inc., 2010.

[2] D. M. Sheen, D. L. McMakin, and T. E. Hall, "Three-dimensional millimeter-wave imaging for concealed weapon detection," IEEE Trans. Microw. Theory Tech., vol. 49, no. 9, pp. 1581-1592, Sep. 2001.

[3] S. Kharkovsky and R. Zoughi, "Microwave and millimeter wave nondestructive testing and evaluation - overview and recent advances," IEEE Instrum. Meas. Mag., vol. 10, no. 2, pp. 26-38, April 2007.

[4] J. Laviada, Y. Álvarez, A. Arboleya, F. Las-Heras, and B. GonzálezValdés, "Multiview techniques for mm-wave imaging," in IEEE International Symposium on Antennas and Propagation, San Diego, USA, 9-14 July, 2017, pp. 1507-1508.

[5] R. Hartley and A. Zisserman, Multiple View Geometry in Computer Vision. Cambridge University Press, 2003.

[6] J. Laviada, A. Arboleya, Y. Álvarez, B. González-Valdés, and F. LasHeras, "Multiview three-dimensional reconstruction by millimetre-wave portable camera," Scientific Reports, vol. 7, no. 6479, 2017.

[7] M. T. Ghasr, M. J. Horst, M. R. Dvorsky, and R. Zoughi, "Wideband microwave camera for real-time 3-D imaging," IEEE Trans. Antennas Propag., vol. 65, no. 1, pp. 258-268, Jan. 2017.

[8] J. M. Lopez-Sanchez and J. Fortuny-Guasch, "3-D radar imaging using range migration techniques," IEEE Trans. Antennas Propag., vol. 48, no. 5, pp. 728-737, May 2000. 